ITEP-TH-1/04

\title{
Representation theory and quantum integrability *
}

\author{
A. Gerasimov ${ }^{\dagger \ddagger}$ S. Kharchev ${ }^{\S}$ D. Lebedev ${ }^{\S}$
}

\begin{abstract}
We describe new constructions of the infinite-dimensional representations of $U(\mathfrak{g})$ and $U_{q}(\mathfrak{g})$ for $\mathfrak{g}$ being $\mathfrak{g l}(N)$ and $\mathfrak{s l}(N)$. The application of these constructions to the quantum integrable theories of Toda type is discussed. With the help of these infinitedimensional representations we manage to establish direct connection between group theoretical approach to the quantum integrability and Quantum Inverse Scattering Method based on the representation theory of Yangian and its generalizations.

In the case of $U_{q}(\mathfrak{g})$ the considered representation is naturally supplied with the structure of $U_{q}(\mathfrak{g}) \otimes U_{\tilde{q}}(\check{\mathfrak{g}})$-bimodule where $\check{\mathfrak{g}}$ is Langlands dual to $\mathfrak{g}$ and $\log q / 2 \pi i=$ $-(\log \tilde{q} / 2 \pi i)^{-1}$. This bimodule structure is a manifestation of the Morita equivalence of the algebra and its dual.
\end{abstract}

\footnotetext{
*Extended talk by the third author at the satellite XIV ICMP workshop: "Infinite Dimensional Algebras and Quantum Integrable systems", July 21-25, 2003, Faro, Portugal

${ }^{\dagger}$ Institute for Theoretical and Experimental Physics, Moscow, Russia

${ }^{\ddagger}$ Hamilton Mathematical Institute at Trinity College, Dublin, Ireland

$\S$ Institute for Theoretical and Experimental Physics, Moscow, Russia

IInstitute for Theoretical and Experimental Physics, Moscow, Russia
} 


\section{Contents}

\begin{tabular}{llr}
\hline & Introduction & 2
\end{tabular}

2 Whittaker modules in the Gelfand-Zetlin representation 4

2.1 The representation of $U(\mathfrak{a l}(N)] \ldots \ldots$. . . . . . . . . . . . . 4

2.2 Whittaker modules . . . . . . . . . . . . . . . . . . 5

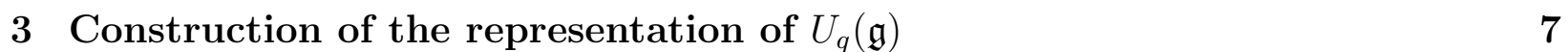

3.1 The rational forms of $U_{q}(\mathfrak{a}) \ldots \ldots \ldots$. . . . . . . . . . . . 8

$3.2 U_{q}(\mathfrak{q})$ in terms of the quantum tori $\ldots \ldots \ldots$. . . . . . . . . . . 9

$3.3 U_{q}(\mathfrak{q}) \otimes U_{\tilde{q}}(\check{\mathfrak{q}})$-bimodule structure $\ldots \ldots \ldots \ldots$

3.4 Whittaker modules . . . . . . . . . . . . . . . . . . 14

4 The QISM's eigenfunction via representation theory 16

4.1 An infinite dimensional representations of the Yangian . . . . . . . . . 16

4.2 The Toda chains and $R$-matrix formalism . . . . . . . . . . . . . . . . . . . . 19

4.3 The spectral problem for the open Toda chain . . . . . . . . . . . . . . . 21

\section{Introduction}

Since the early days of quantum mechanics, the representation theory plays the important role as a succinct tool to describe and explicitly solve quantum theories. On the other hand, the problems in quantum theories serve as a source of the new ideas in representation theory. The most notable recent example is the emergence of the notion of the quantum groups [1], 2] from the algebraic formulation of the Quantum Inverse Scattering Method (QISM) 3], [4].

In this paper we describe several constructions in representation theory of classical and quantum groups inspired by our studies of the simple quantum integrable models. Our starting point was the desire to understand QISM in more standard representation theory terms. It was a surprise that one should instead invent the new constructions in the representation theory. It is well known to experts in integrable systems that there exist distinguished coordinates in which the description of the quantum system, although non trivial, is drastically simplified. Multi-dimensional nonlinear spectral problems are reduced to the one dimensional case and the solution of the quantum theory may be constructed explicitly. The recent interest in this approach initiated by Sklyanin [5] leads to an explosion of activity connected with a search of the explicit transforms to such coordinates. This Separation of Variables method (SoV) may be considered as an extension/generalization of QISM.

It was shown in [6], 7] that the separation of variables in the modern form has a clear group theoretic meaning and goes back to the natural parameterization of the regular group 
elements of the non-compact groups. This leads to the new construction of the representations of the universal enveloping of the Lie algebras in terms of the difference operators. A certain particular case of this construction leading to the finite dimensional representations turns out to be the well known Gelfand-Zetlin recursive construction of the representations of classical groups [8], 9], 10].

The explicit connection with the QISM approach appears through the closely related construction of the representations of the Yangian. Namely, let $\mathfrak{g}$ be the Lie algebra $\mathfrak{g l}(N)$ and let $Y(\mathfrak{g})$ be the Yangian [11]. There is a natural epimorphism $\pi_{N}: Y(\mathfrak{g}) \rightarrow \mathcal{U}(\mathfrak{g})$ compatible with the representation in terms of the difference operators. The constructed representation of the Yangian turns out to be a manifestation of the simultaneous existence of the RTT-type realization and Drinfeld's "new" realization [12. The distinguished maximal commutative subalgebra of the Yangian is a key ingredient in the explicit connection between these two constructions and may be described by the set of the commuting operators $\mathcal{A}_{n}(\lambda), n=$ $1, \ldots, N$. In the special class of the representations $\mathcal{A}_{n}(\lambda)$ act as polynomials in $\lambda$. The zeros of these polynomials provide the variables appearing in SoV. On the other hand, these variables are exactly the variables that appear in our generalization of Gelfand-Zetlin construction. Note that Drinfeld's "new" realization is known for the Yangian of an arbitrary simple Lie algebra and the generalized Gelfand-Zetlin construction of the representation of $\mathfrak{g l}(N)$ may be naturally extended to the case of the general simple Lie algebra.

There is a further generalization of the construction providing the explicit infinitedimensional representation of the quantum group $U_{q}(\mathfrak{g})$ for $\mathfrak{g}=\mathfrak{g l}(N), \mathfrak{s l}(N)$. However, a new essential phenomenon appears in this case. The infinite-dimensional representations of $U_{q}(\mathfrak{g})$ are naturally supplied with the structure of $U_{q}(\mathfrak{g}) \otimes U_{\tilde{q}}(\check{\mathfrak{g}})$-bimodule where $\check{\mathfrak{g}}$ is Langlands dual to $\mathfrak{g}$ and $\log q / 2 \pi i=-(\log \tilde{q} / 2 \pi i)^{-1}$ (for the preliminary results in these direction see [13], 14, [15]). We give explicit construction of this bimodule structure and show the natural appearance of the Langlands dual for $U_{q}(\mathfrak{g})$, when $\mathfrak{g}=\mathfrak{g l}(N), \mathfrak{s l}(N)$. Note that in this paper we essentially use various rational forms of the quantum groups (see [16], 17] and references therein). Thus, the Langlands dual to the minimal ("adjoint") rational form of $U_{q}(\mathfrak{s l}(N))$ turns out to be another form of $U_{\tilde{q}}(\mathfrak{s l}(N))$ in accordance with the fact that the Langlands dual to $P S L(N)$ is $S L(N)$ for the classical groups. Further study shows that the appearance of the Langlands dual in this construction is not accidental and is a manifestation of the Morita equivalence of certain two algebras naturally associated with $U_{q}(\mathfrak{g})$ and $U_{\tilde{q}}(\check{\mathfrak{g}})$ (compare with [18, [19]). This formulation appears to be very close to the initial problem of the classification of the irreducible representations in the decomposition of the regular representation of the group $G$ in terms of the dual group $\check{G}[20$. We are planning to discuss this construction in full details elsewhere.

The plan of the paper is as follows. In section 2 we review the part of [6] concerning the analytic continuation of the Gelfand-Zetlin construction to the case of Whittaker modules of $U(\mathfrak{g l}(N))$. An explicit description of a Whittaker module is straightforward as soon as we have an explicit expression for the cyclic Whittaker vector. In section 3 we generalize this construction to the case of quantum groups. The structure of $U_{q}(\mathfrak{g}) \otimes U_{\tilde{q}}(\check{\mathfrak{g}})$-bimodule and its connection with Langlands duality is shortly discussed. In section 4 we demonstrate the connection with SoV and QISM and describe the applications to the quantum integrable theories of Toda type. 
The results of these paper are based on the work that was done for the past few years by the ITEP group in Moscow and was reported in the series of papers [21, [22], 23], [15], [6], 7].

Acknowledgments: The research was partly supported by grants CRDF RM1-2545MO-03; INTAS 03-513350; grant 1999.2003.2 for support of scientific schools, and by grants RFBR 03-02-17554 (A. Gerasimov, D. Lebedev), RFBR 03-02-17373 (S. Kharchev). We are grateful to M.Kontsevich, and M. Semenov-Tian-Shansky for their interest in this work and we are grateful to A. Rosly for useful discussion. D.L. is also grateful to IHES for warm hospitality and to organizers and participants of the ICMP workshop in Faro for the creation of the stimulating atmosphere. D.L. thanks to 21 COE RIMS Research Project 2004: Quantum Integrable Systems and Infinite Dimensional Algebras, where the paper was finished, for support and warm hospitality.

\section{Whittaker modules in the Gelfand-Zetlin represen- tation}

\subsection{The representation of $U(\mathfrak{g l}(N))$}

Let us remind the construction of the paper [6], where an analytical continuation the GelfandZetlin (GZ) theory to infinite-dimensional representations of the universal enveloping algebra $U(\mathfrak{g l}(N))$ was introduced.

Let $\hat{\mathbb{T}}_{\hbar}$ be an associative algebra generated by $\hat{\gamma}_{n j}, \hat{\beta}_{n j}^{ \pm 1}, n=1, \ldots, N-1 ; 1 \leq j \leq n$, and $\hat{\gamma}_{N j}, 1 \leq j \leq N$, subject to the relations

$$
\begin{gathered}
{\left[\hat{\gamma}_{n j}, \hat{\gamma}_{m l}\right]=\left[\hat{\beta}_{n j}, \hat{\beta}_{m l}\right]=0,} \\
{\left[\hat{\beta}_{m l}, \hat{\gamma}_{n j}\right]=i \hbar \delta_{m n} \delta_{l j} \hat{\beta}_{m l} .}
\end{gathered}
$$

Theorem 2.1 Let $E_{n m}, n, m=1, \ldots, N$ be the generators of $\mathfrak{g l}(N)$. The following explicit expressions defines the embedding $\pi: \mathfrak{g l}(N) \hookrightarrow \hat{\mathbb{T}}_{\hbar}$

$$
\begin{array}{cc}
E_{n n}=\frac{1}{i \hbar}\left(\sum_{j=1}^{n} \hat{\gamma}_{n j}-\sum_{j=1}^{n-1} \hat{\gamma}_{n-1, j}\right), & (n=1, \ldots, N), \\
E_{n, n+1}=-\frac{1}{i \hbar} \sum_{j=1}^{n} \frac{\prod_{r=1}^{n+1}\left(\hat{\gamma}_{n j}-\hat{\gamma}_{n+1, r}-\frac{i \hbar}{2}\right)}{\prod_{s \neq j}\left(\hat{\gamma}_{n j}-\hat{\gamma}_{n s}\right)} \hat{\beta}_{n j}^{-1}, \quad(n=1, \ldots, N-1), \\
E_{n+1, n}=\frac{1}{i \hbar} \sum_{j=1}^{n} \frac{\prod_{r=1}^{n-1}\left(\hat{\gamma}_{n j}-\hat{\gamma}_{n-1, r}+\frac{i \hbar}{2}\right)}{\prod_{s \neq j}\left(\hat{\gamma}_{n j}-\hat{\gamma}_{n s}\right)} \hat{\beta}_{n j}, \quad(n=1, \ldots, N-1) .
\end{array}
$$


Let us consider the following natural representation of the quantum torus $\hat{\mathbb{T}}_{\hbar}$ :

$$
\begin{aligned}
& \hat{\gamma}_{n j}=\gamma_{n j} \in \mathbb{C}, \quad n=1, \ldots, N, \quad 1 \leq j \leq n, \\
& \hat{\beta}_{n j}=e^{i \hbar \frac{\partial}{\partial \gamma_{n j}}}, \quad n=1, \ldots, N-1, \quad 1 \leq j \leq n,
\end{aligned}
$$

where $\gamma_{n j}$ and $e^{ \pm i \hbar \frac{\partial}{\partial \gamma_{n j}}}$ are considered as operators acting on the space $M$ of meromorphic functions of complex variables $\gamma_{n j}, n=1, \ldots, N-1,1 \leq j \leq n$. The remaining $\gamma_{N 1}, \ldots, \gamma_{N N}$ are considered as constants. Thus the complex vector $\gamma_{N}=\left(\gamma_{N 1}, \ldots, \gamma_{N N}\right)$ plays the role of a label which determines the above representation.

Let $\mathcal{Z}(\mathfrak{g l}(n))$ be the center of $U(\mathfrak{g l}(n))$. We say that a $U(\mathfrak{g l}(n))$-module $V$ admits an infinitesimal character $\xi$ if there is a homomorphism $\xi: \mathcal{Z}(\mathfrak{g l}(n)) \rightarrow \mathbb{C}$ such that $z v=\xi(z) v$ for all $z \in \mathcal{Z}(\mathfrak{g l}(n)), v \in V$. It is possible to show that the $U(\mathfrak{g l}(N))$-module $M$ defined above admits an infinitesimal character and each central element of $U(\mathfrak{g l}(n))$ acts on $M$ via multiplication by a symmetric polynomial in the variables $\gamma_{n j}$ [6]

In the next subsection we calculate the explicit action of the central elements on $M$ using the notion of Whittaker vectors.

\subsection{Whittaker modules}

Let us now give a construction for Whittaker modules using the representation of $U(\mathfrak{g l}(N))$ described above. We first recall some facts from [24].

Let $n_{+}$and $n_{-}$be the subalgebras of $\mathfrak{g l}(N)$ generated, respectively, by positive and negative root generators. The homomorphisms (characters) $\chi_{+}: n_{+} \rightarrow \mathbb{C}, \chi_{-}: n_{-} \rightarrow \mathbb{C}$ are uniquely determined by their values on the simple root generators, and are called non-singular if the (complex) numbers $\chi_{+}\left(E_{n, n+1}\right)$ and $\chi_{-}\left(E_{n+1, n}\right)$ are non-zero for all $n=1, \ldots, N-1$.

Let $V$ be any $U=U(\mathfrak{g l}(N))$-module. Denote the action of $u \in U$ on $v \in V$ by $u v$. A vector $w \in V$ is called a Whittaker vector with respect to the character $\chi_{+}$if

$$
E_{n, n+1} w=\chi_{+}\left(E_{n, n+1}\right) w, \quad(n=1, \ldots, N-1)
$$

and an element $w^{\prime} \in V^{\prime}$ is called a Whittaker vector with respect to the character $\chi_{-}$if

$$
E_{n+1, n} w^{\prime}=\chi_{-}\left(E_{n+1, n}\right) w^{\prime}, \quad(n=1, \ldots, N-1) .
$$

A Whittaker vector is cyclic for $V$ if $U w=V$, and a $U$-module is a Whittaker module if it contains a cyclic Whittaker vector. The $U$-modules $V$ and $V^{\prime}$ are called dual if there exists a non-degenerate pairing $\langle.,\rangle:. V^{\prime} \times V \rightarrow \mathbb{C}$ such that $\left\langle X v^{\prime}, v\right\rangle=-\left\langle v^{\prime}, X v\right\rangle$ for all $v \in V, v^{\prime} \in V^{\prime}$ and $X \in \mathfrak{g l}(N)$.

We proceed with explicit formulas for Whittaker vectors corresponding to the representation given by (2.2), (2.3).

Proposition 2.1 The equations

$$
E_{n+1, n} w_{N}^{\prime}=-i \hbar^{-1} w_{N}^{\prime},
$$




$$
E_{n, n+1} w_{N}=-i \hbar^{-1} w_{N}
$$

for all $n=1, \ldots, N-1$, admit the solutions

$$
\begin{gathered}
w_{N}^{\prime}=1 \\
w_{N}=e^{-\frac{\pi}{\hbar} \sum_{n=1}^{N-1}(n-1) \sum_{j=1}^{n} \gamma_{n j}} \prod_{n=1}^{N-1} s_{n}\left(\boldsymbol{\gamma}_{n}, \gamma_{n+1}\right),
\end{gathered}
$$

where

$$
s_{n}\left(\gamma_{n}, \gamma_{n+1}\right)=\prod_{k=1}^{n} \prod_{m=1}^{n+1} \hbar^{\frac{\gamma_{n k}-\gamma_{n+1, m}}{i \hbar}+\frac{1}{2}} \Gamma\left(\frac{\gamma_{n k}-\gamma_{n+1, m}}{i \hbar}+\frac{1}{2}\right)
$$

( For the proof see [6].)

The solutions (2.8), (2.9) are not unique. Indeed, the set of Whittaker vectors is closed under the multiplication by an arbitrary $i \hbar$-periodic function in the variables $\gamma_{n j}$. Hence, there are infinitely many invariant subspaces in $M$ corresponding to infinitely many Whittaker vectors.

To construct irreducible submodules, let us introduce the Whittaker modules $W$ and $W^{\prime}$, generated cyclically by the Whittaker vectors $w_{N}$ and $w_{N}^{\prime}$, respectively. An explicit description of a Whittaker module is straightforward as soon as we have an explicit expression for the Whittaker vectors. Namely let $\boldsymbol{m}_{n}=\left(m_{n 1}, \ldots, m_{n n}\right)$ be the set of non-negative integers. The Whittaker module $W=U w_{N}$ is spanned by the elements

$$
w_{\boldsymbol{m}_{1}, \ldots, \boldsymbol{m}_{N-1}}=\prod_{n=1}^{N-1} \prod_{k=1}^{n} \sigma_{k}^{m_{n k}}\left(\boldsymbol{\gamma}_{n}\right) w_{N}
$$

where $\sigma_{k}\left(\boldsymbol{\gamma}_{n}\right)$ is the elementary symmetric function of the variables $\gamma_{n 1}, \ldots, \gamma_{n n}$ of order $k$ :

$$
\sigma_{k}\left(\gamma_{n}\right)=\sum_{j_{1}<\ldots<j_{k}} \gamma_{n j_{1}} \ldots \gamma_{n j_{k}}
$$

Similarly, the Whittaker module $W^{\prime}=U w_{N}^{\prime}$ is spanned by the polynomials

$$
w_{\boldsymbol{m}_{1}, \ldots, \boldsymbol{m}_{N-1}}^{\prime}=\prod_{n=1}^{N-1} \prod_{k=1}^{n} \sigma_{k}^{m_{n k}}\left(\boldsymbol{\gamma}_{n}\right)
$$

The Whittaker modules $W$ and $W^{\prime}$ are irreducible.

Let us note that for any subalgebra $U(\mathfrak{g l}(n)) \subset U(\mathfrak{g l}(N)), 2 \leq n<N$, the module over the ring of the polynomials in $\gamma_{n}$ with the basis $\prod_{l=1}^{n-1} \prod_{k=1}^{l} \sigma_{k}^{m_{l k}}\left(\boldsymbol{\gamma}_{l}\right) w_{N}$ is a $U(\mathfrak{g l}(n))$ Whittaker module. One can calculate the explicit form of the action of the central elements of $U(\mathfrak{g l}(n))$ 
on the space $M$. It is well known that the generating function $\mathcal{A}_{n}(\lambda)$ of the central elements of $U(\mathfrak{g l}(n))$ (the Casimir operators) can be represented as follows [25]:

$$
\begin{gathered}
\mathcal{A}_{n}(\lambda) \\
=\sum_{s \in S_{n}} \operatorname{sign} s\left[\left(\lambda-i \hbar \rho_{1}^{(n)}\right) \delta_{s(1), 1}-i \hbar E_{s(1), 1}\right] \ldots\left[\left(\lambda-i \hbar \rho_{n}^{(n)}\right) \delta_{s(n), n}-i \hbar E_{s(n), n}\right],
\end{gathered}
$$

where $\rho_{k}^{(n)}=\frac{1}{2}(n-2 k+1), \quad k=1, \ldots, n$ and the summation is over elements of the permutation group $S_{n}$. It can be proved [6] that the operators (2.13) have the following form on a space of meromorphic functions $M$ :

$$
\mathcal{A}_{n}(\lambda)=\prod_{j=1}^{n}\left(\lambda-\gamma_{n j}\right), \quad(n=1, \ldots, N) .
$$

It remains to construct a pairing between $W$ and $W^{\prime}$, and to prove that the Whittaker modules $W$ and $W^{\prime}$ are dual with respect to this pairing. Let $\phi \in W^{\prime}$ and $\psi \in W$. Define the pairing $\langle.,\rangle:. W^{\prime} \otimes W \rightarrow \mathbb{C}$ by

$$
\langle\phi, \psi\rangle=\int_{\mathbb{R}^{\frac{N(N-1)}{2}}} \mu_{0}(\gamma) \bar{\phi}(\gamma) \psi(\gamma) \prod_{\substack{n=1 \\ j \leq n}}^{N-1} d \gamma_{n j}
$$

where

$$
\mu_{0}(\gamma)=\prod_{n=2}^{N-1} \prod_{p<r}\left(\gamma_{n p}-\gamma_{n r}\right)\left(e^{\frac{2 \pi \gamma_{n p}}{\hbar}}-e^{\frac{2 \pi \gamma_{n n}}{\hbar}}\right)
$$

The integral (2.15) converges absolutely (See for the proof [6]).

To construct the pair of the dual Whittaker modules, we should restrict the label of representation to the real values: $\gamma_{N} \in \mathbb{R}^{N}$. Then the Whittaker modules $W$ and $W^{\prime}$ will be dual with respect to the pairing defined by (2.15). That is for any $\phi \in W^{\prime}$ and $\psi \in W$, the generators $X \in \mathfrak{g l}(N, \mathbb{R})$ possess the property

$$
\langle\phi, X \psi\rangle=-\langle X \phi, \psi\rangle \text {. }
$$

This property will be important in the derivation of the wave function in Section 4 .

\section{Construction of the representation of $U_{q}(\mathfrak{g})$}

In this section we outline an extension of our approach to the quantum groups. We start with the construction of the embedding of $U_{q}(\mathfrak{g})$ in the product of a commutative and noncommutative tori. The skew field of fractions constructed from $U_{q}(\mathfrak{g})$ coincides with the functions on the product of the tori invariant under the product of the symmetric groups. Then we describe explicitly the structure of $U_{q}(\mathfrak{g}) \otimes U_{\tilde{q}}(\check{\mathfrak{g}})$ bimodule. 


\subsection{The rational forms of $U_{q}(\mathfrak{g})$}

We start with the definition of the quantum groups following [16, [17. Let $q$ be an indeterminate, and let $\mathbb{C}(q)$ be the field of rational functions of $q$ with coefficients in $\mathbb{C}$. The associative $\mathbb{C}(q)$-algebra $U_{q}(\mathfrak{g l}(N))$ is generated by the elements $K_{n n}^{ \pm 1}, E_{n m} ; n \neq m ; n, m=1, \ldots, N$ subjected to the relations ${ }^{1}$ :

$$
\begin{gathered}
K_{n n} K_{n n}^{-1}=K_{n n}^{-1} K_{n n}=1, \quad K_{n n} K_{m m}=K_{m m} K_{n n}, \\
K_{n n} E_{m, m+1} K_{n n}^{-1}=q^{\delta_{n m}-\delta_{n, m+1}} E_{m, m+1}, \\
K_{n n} E_{m+1, m} K_{n n}^{-1}=q^{\delta_{n, m+1}-\delta_{n m}} E_{m+1, m}, \\
E_{n, n+1} E_{m+1, m}-E_{m+1, m} E_{n, n+1}=\delta_{n m} \frac{K_{n n} K_{n+1, n+1}^{-1}-K_{n n}^{-1} K_{n+1, n+1}}{q-q^{-1}}
\end{gathered}
$$

together with quantum analogues of Serre relations

$$
\begin{gathered}
E_{n, n+1} E_{m, m+1}-E_{m, m+1} E_{n, n+1}=0, \quad m \neq n \pm 1, \\
E_{n, n+1}^{2} E_{n+1, n+2}-\left(q+q^{-1}\right) E_{n, n+1} E_{n+1, n+2} E_{n, n+1}+E_{n+1, n+2} E_{n, n+1}^{2}=0, \\
E_{n+1, n+2}^{2} E_{n, n+1}-\left(q+q^{-1}\right) E_{n+1, n+2} E_{n, n+1} E_{n+1, n+2}+E_{n, n+1} E_{n+1, n+2}^{2}=0, \\
E_{n+1, n} E_{m+1, m}-E_{m+1, m} E_{n+1, n}=0, \quad m \neq n \pm 1, \\
E_{n+1, n}^{2} E_{n+2, n+1}-\left(q+q^{-1}\right) E_{n+1, n} E_{n+2, n+1} E_{n+1, n}+E_{n+2, n+1} E_{n+1, n}^{2}=0, \\
E_{n+2, n+1}^{2} E_{n+1, n}-\left(q+q^{-1}\right) E_{n+2, n+1} E_{n+1, n} E_{n+2, n+1}+E_{n+1, n} E_{n+2, n+1}^{2}=0 .
\end{gathered}
$$

We will also need the explicit description of the quantum group $U_{q}(\mathfrak{s l}(N))$. Note that there exist various rational forms of quantum groups [16], [17. In the case of $U_{q}(\mathfrak{s l}(N))$ we have the following description. Let $a_{n m}=2 \delta_{n m}-\delta_{n, m+1}-\delta_{n+1, n}$ be the Cartan matrix of $\mathfrak{s l}(N)$. Let $Q$ and $P$ denote the root lattice and the lattice of weights, respectively. The smallest rational form, the adjoint rational form $U_{q}^{Q}(\mathfrak{s l}(N))$, is the associative $\mathbb{C}(q)$-algebra with generators $E_{n}, F_{n}$ and $K_{n}^{ \pm 1}, n=1, \ldots, N-1$, and relations

$$
\begin{gathered}
K_{n} K_{n}^{-1}=K_{n}^{-1} K_{n}=1, \quad K_{n} K_{m}=K_{m} K_{n}, \\
K_{n} E_{m} K_{n}^{-1}=q^{a_{n m}} E_{m}, \quad K_{n} F_{m} K_{n}^{-1}=q^{-a_{n m}} F_{m}, \\
E_{n} F_{m}-F_{m} E_{n}=\delta_{n m} \frac{K_{n}-K_{n}^{-1}}{q-q^{-1}},
\end{gathered}
$$

\footnotetext{
${ }^{1}$ We will not use the Hopf structure of the quantum groups and, therefore, we omit the comultiplication formulas in what follows.
} 


$$
\begin{aligned}
& \sum_{r=0}^{1-a_{n m}}(-1)^{r}\left[\begin{array}{c}
1-a_{n m} \\
r
\end{array}\right]_{q} E_{n}^{1-a_{n m}-r} E_{m} E_{n}^{r}=0, \quad \text { if } n \neq m, \\
& \sum_{r=0}^{1-a_{n m}}(-1)^{r}\left[\begin{array}{c}
1-a_{n m} \\
r
\end{array}\right]_{q} F_{n}^{1-a_{n m}-r} F_{m} F_{n}^{r}=0, \quad \text { if } n \neq m .
\end{aligned}
$$

Here we have used the standard notations

$$
\left[\begin{array}{c}
m \\
n
\end{array}\right]_{q}=\frac{[m]_{q} !}{[n]_{q} ![m-n]_{q} !}, \quad[m]_{q} !=\prod_{1 \leq j \leq m} \frac{q^{j}-q^{-j}}{q-q^{-1}} .
$$

The largest $\mathbb{C}(q)$-algebra, the simply-connected rational form $U_{q}^{P}(\mathfrak{s l}(N))$, is obtained by adjoining to $U_{q}^{Q}(\mathfrak{s l}(N))$ the invertible elements $L_{n}, n=1 \ldots, N-1$, such that $K_{n}=\prod_{m} L_{m}^{a_{m n}}$. The relations (3.3b) are now replaced by

$$
L_{n} E_{m} L_{n}^{-1}=q^{\delta_{n m}} E_{m}, \quad L_{n} F_{m} L_{n}^{-1}=q^{-\delta_{n m}} F_{m} .
$$

Denote by $M$ any lattice such that $Q \subseteq M \subseteq P$. A general rational form $U_{q}^{M}(\mathfrak{g})$ is obtained by adjoining to $U_{q}^{Q}(\mathfrak{g})$ the elements $K_{\beta_{i}}=\prod_{j} L_{j}^{m_{i j}}$ for every $\beta_{i}=\sum_{j} m_{i j} \lambda_{j} \in M$, where $\lambda_{j}$ are the fundamental weights. Note that various rational forms $U_{q}^{M}$ have interesting applications to the construction of quantum integrable systems of Toda type.

\section{2 $U_{q}(\mathfrak{g})$ in terms of the quantum tori}

We start with the definition of quantum torus algebra $\hat{\mathbb{T}}_{q}$. Let $\hat{\mathbb{T}}_{q}$ be the associative $\mathbb{C}(q)$ algebra of the rational functions of invertible elements $\mathbf{v}_{n j}, n=1, \ldots, N ; j=1, \ldots, n$ and $\mathbf{u}_{n j}, n=1, \ldots, N-1 ; j=1, \ldots, n$ which are subjected to the relations

$$
\begin{gathered}
\mathbf{v}_{n j} \mathbf{v}_{m k}=\mathbf{v}_{m k} \mathbf{v}_{n j}, \quad \mathbf{u}_{n j} \mathbf{u}_{m k}=\mathbf{u}_{m k} \mathbf{u}_{n j}, \\
\mathbf{u}_{n j} \mathbf{v}_{m k}=q^{\delta_{n m} \delta_{j k}} \mathbf{v}_{m k} \mathbf{u}_{n j} .
\end{gathered}
$$

Theorem 3.1 (i) Let $K_{n n}^{ \pm 1}, E_{n m}$ be the generators of $U_{q}(\mathfrak{g l}(N))$. The following explicit expressions define the embedding $\pi: U_{q}(\mathfrak{g l}(N)) \hookrightarrow \hat{\mathbb{T}}_{q}$

$$
\begin{gathered}
\pi\left(K_{n n}\right)=\prod_{j=1}^{n} \mathbf{v}_{n j} \prod_{j=1}^{n-1} \mathbf{v}_{n-1, j}^{-1}, \\
\pi\left(E_{n, n+1}\right)=-\frac{q^{-1}}{q-q^{-1}} \prod_{j=1}^{n+1} \mathbf{v}_{n+1, j}^{-1} \prod_{j=1}^{n} \mathbf{v}_{n, j} \sum_{j=1}^{n} \mathbf{v}_{n j}^{-3} \frac{\prod_{r=1}^{n+1}\left(\mathbf{v}_{n j}^{2}-q \mathbf{v}_{n+1, r}^{2}\right)}{\prod_{s \neq j}\left(\mathbf{v}_{n j}^{2}-\mathbf{v}_{n s}^{2}\right)} \mathbf{u}_{n j}^{-1}, \\
\pi\left(E_{n+1, n}\right)=\frac{1}{q-q^{-1}} \prod_{j=1}^{n} \mathbf{v}_{n j} \prod_{j=1}^{n-1} \mathbf{v}_{n-1, j}^{-1} \sum_{j=1}^{n} \mathbf{v}_{n j}^{-1} \frac{\prod_{r=1}^{n-1}\left(\mathbf{v}_{n j}^{2}-q^{-1} \mathbf{v}_{n-1, r}^{2}\right)}{\prod_{s \neq j}\left(\mathbf{v}_{n j}^{2}-\mathbf{v}_{n s}^{2}\right)} \mathbf{u}_{n j} .
\end{gathered}
$$


(ii) Let $L_{n}^{ \pm 1}, K_{n}^{ \pm 1}, E_{n}, F_{n}$ be the generators of $U_{q}(\mathfrak{s l}(N))$. The following explicit expressions define the embedding $\pi: U_{q}(\mathfrak{s l}(N)) \hookrightarrow \hat{\mathbb{T}}_{q}$ :

$$
\begin{gathered}
\pi\left(L_{n}\right)=\prod_{j=1}^{n} \mathbf{v}_{n j}, \quad \prod_{j=1}^{N} \mathbf{v}_{N j}=1 \\
\pi\left(K_{n}\right)=\prod_{m}\left(\prod_{j=1}^{m} \mathbf{v}_{m j}\right)^{a_{m n}}, \\
\pi\left(E_{n}\right)=-\frac{q^{-1}}{q-q^{-1}} \prod_{j=1}^{n+1} \mathbf{v}_{n+1, j}^{-1} \prod_{j=1}^{n} \mathbf{v}_{n j} \sum_{j=1}^{n} \mathbf{v}_{n j}^{-3} \frac{\prod_{r=1}^{n+1}\left(\mathbf{v}_{n j}^{2}-q \mathbf{v}_{n+1, r}^{2}\right)}{\prod_{s \neq j}\left(\mathbf{v}_{n j}^{2}-\mathbf{v}_{n s}^{2}\right)} \mathbf{u}_{n j}^{-1} \\
\pi\left(F_{n}\right)=\frac{1}{q-q^{-1}} \prod_{j=1}^{n} \mathbf{v}_{n j} \prod_{j=1}^{n-1} \mathbf{v}_{n-1, j}^{-1} \sum_{j=1}^{n} \mathbf{v}_{n j}^{-1} \frac{\prod_{r=1}^{n-1}\left(\mathbf{v}_{n j}^{2}-q^{-1} \mathbf{v}_{n-1, r}^{2}\right)}{\prod_{s \neq j}\left(\mathbf{v}_{n j}^{2}-\mathbf{v}_{n s}^{2}\right)} \mathbf{u}_{n j} .
\end{gathered}
$$

The algebra $\hat{\mathbb{T}}_{q}$ is very close to the minimal skew field of fractions of $U_{q}(\mathfrak{g l}(N))$ for which the inclusion of the universal enveloping algebra is possible. Consider the skew field of fractions $\mathcal{D}\left(U_{q}(\mathfrak{g})\right)$ of $U_{q}(\mathfrak{g})$ which consists of the elements of the form $u \cdot v^{-1}$ or $x^{-1} \cdot y$, where $u, v, x, y \in U_{q}(\mathfrak{g})$ (for more details see e.g. [26]). It appears that the skew field of fractions $\mathcal{D}\left(U_{q}(\mathfrak{g l}(N))\right)$ is obtained by partial symmetrization of the algebra $\hat{\mathbb{T}}_{q}$

$$
\mathcal{D}\left(U_{q}(\mathfrak{g l}(N))\right)=\left(\hat{\mathbb{T}}_{q}\right)^{\otimes_{n=1}^{N} S_{n}}
$$

where, for $n<N$, the group $S_{n}$ acts as:

$$
\begin{aligned}
& \sigma: \mathbf{v}_{n j} \rightarrow \mathbf{v}_{n, \sigma(j)} \\
& \sigma: \mathbf{u}_{n j} \rightarrow \mathbf{u}_{n, \sigma(j)}
\end{aligned}
$$

and the group $S_{N}$ acts as:

$$
\sigma: \mathbf{v}_{N j} \rightarrow \mathbf{v}_{N, \sigma(j)}
$$

This proposition can be considered as a generalization of the Gelfand-Kirillov theorem for $U(\mathfrak{g})[26]$.

\section{3 $U_{q}(\mathfrak{g}) \otimes U_{\tilde{q}}(\check{\mathfrak{g}})$-bimodule structure}

It turns out that the consideration of the infinite-dimensional representation of various rational forms (3.3)-(3.7) of quantum groups reveals new phenomena. Namely some representations of the quantum group $U_{q}(\mathfrak{g})$ poses a natural structure of a $U_{q}(\mathfrak{g}) \otimes U_{\tilde{q}}(\check{\mathfrak{g}})$-bimodule 
where $\check{\mathfrak{g}}$ is Langlands dual to $\mathfrak{g}$ and $\log q / 2 \pi i=-(\log \tilde{q} / 2 \pi i)^{-1}$. The key point is the interpretation of the appropriately defined centralizer of the image of $U_{q}(\mathfrak{g})$ in these representations in terms of the representation of $U_{\tilde{q}}(\check{\mathfrak{g}})$. The presence of the Langlands dual will be verified below in the case of $U_{q}(\mathfrak{s l}(N))$. It turns out that the centralizer construction leads to the connection between various forms of $U_{q}(\mathfrak{s l}(N))$. In particular, when we start from the minimal (adjoint) form, we come to what may be called the maximal form over the quadratic extension, which is in agreement with the classical picture of the duality between $S L(N)$ and $\operatorname{PSL}(N)$.

Let us begin with the construction of a representation of $U_{q}(\mathfrak{g l}(N))$ generalizing the representation of $U(\mathfrak{g l}(N))$ described in Section 2. Let $\mathcal{V}$ be the space of functions of $\gamma_{n j}$ with $n=1, \ldots, N-1 ; 1 \leq j \leq n$ and $\mathcal{V}^{s}$ be the space of functions of $\gamma_{n j}$ invariant under the action of $\otimes_{n=1}^{N-1} S_{n}$ according to (3.14a). Let $\mathcal{R}$ be the algebra of rational functions of the exponents of the linear functions of $\gamma_{n j}$ with $n=1, \ldots N ; 1 \leq j \leq n$ and $\partial_{\gamma_{n j}}:=\frac{\partial}{\partial \gamma_{n j}}$ with $n=1, \ldots N-1 ; 1 \leq j \leq n$ and $\mathcal{R}^{s}$ be the algebra of rational functions of the exponents of the linear functions of $\gamma_{n j}$ and $\partial_{\gamma_{n j}}$ invariant under the action of $\otimes_{n=1}^{N} S_{n}$ according to (3.14), (3.15). One has the following embedding $\varrho: \hat{\mathbb{T}}_{q} \hookrightarrow \mathcal{R}$

$$
\varrho\left(\mathbf{u}_{n j}\right)=e^{i \omega_{1} \partial_{\gamma_{n j}}}, \quad \varrho\left(\mathbf{v}_{n j}\right)=e^{\frac{2 \pi \gamma_{n j}}{\omega_{2}}}, \quad \varrho(q)=e^{\frac{2 \pi i \omega_{1}}{\omega_{2}}} .
$$

Using Theorem 3.1 we obtain the representation of $U_{q}(\mathfrak{g l}(N))$ in terms of the difference operators. By an abuse of notation we shall also denote by $\varrho$ the composition $\varrho \circ \pi$.

Proposition 3.1 The following expressions define a representation of $U_{q}(\mathfrak{g l}(N))$ with $q=$ $e^{\frac{2 \pi i \omega_{1}}{\omega_{2}}}$ :

$$
\begin{gathered}
\varrho\left(K_{n n}\right)=e^{\frac{2 \pi}{\omega_{2}}\left(\sum_{j=1}^{n} \gamma_{n j}-\sum_{j=1}^{n-1} \gamma_{n-1, j}\right)} \\
\varrho\left(E_{n, n+1}\right)=\frac{2 i e^{\frac{\pi i \omega_{1}}{\omega_{2}}(n-1)}}{\sin \frac{2 \pi \omega_{1}}{\omega_{2}}} \sum_{j=1}^{n} \frac{\prod_{r=1}^{n+1} \sinh \frac{2 \pi}{\omega_{2}}\left(\gamma_{n j}-\gamma_{n+1, r}-\frac{i \omega_{1}}{2}\right)}{\prod_{s \neq j} \sinh \frac{2 \pi}{\omega_{2}}\left(\gamma_{n j}-\gamma_{n s}\right)} e^{-i \omega_{1} \partial_{\gamma_{n j}}}, \\
\varrho\left(E_{n+1, n}\right)=-\frac{i e^{-\frac{\pi i \omega_{1}}{\omega_{2}}(n-1)}}{2 \sin \frac{2 \pi \omega_{1}}{\omega_{2}}} \sum_{j=1}^{n} \frac{\prod_{r=1}^{n-1} \sinh \frac{2 \pi}{\omega_{2}}\left(\gamma_{n j}-\gamma_{n-1, r}+\frac{i \omega_{1}}{2}\right)}{\prod_{s \neq j} \sinh \frac{2 \pi}{\omega_{2}}\left(\gamma_{n j}-\gamma_{n s}\right)} e^{i \omega_{1} \partial_{\gamma_{n j}}} .
\end{gathered}
$$

Consider the dual quantum torus $\hat{\mathbb{T}}_{\tilde{q}}$ using the invertible elements $\tilde{\mathbf{v}}_{n j}, n=1, \ldots, N ; j=$ $1, \ldots, n$ and $\tilde{\mathbf{u}}_{n j}, n=1, \ldots, N-1 ; j=1, \ldots, n$ subjected to the relations

$$
\begin{gathered}
\tilde{\mathbf{v}}_{n j} \tilde{\mathbf{v}}_{m k}=\tilde{\mathbf{v}}_{m k} \tilde{\mathbf{v}}_{n j}, \quad \tilde{\mathbf{u}}_{n j} \tilde{\mathbf{u}}_{m k}=\tilde{\mathbf{u}}_{m k} \tilde{\mathbf{u}}_{n j}, \\
\tilde{\mathbf{u}}_{n j} \tilde{\mathbf{v}}_{m k}=\tilde{q}^{\delta_{n m} \delta_{j k}} \tilde{\mathbf{v}}_{m k} \tilde{\mathbf{u}}_{n j} .
\end{gathered}
$$

One has the dual embedding $\tilde{\varrho}: \hat{\mathbb{T}}_{\tilde{q}} \hookrightarrow \mathcal{R}$

$$
\tilde{\varrho}\left(\tilde{\mathbf{u}}_{n j}\right)=e^{i \omega_{2} \partial_{\gamma_{n j}}}, \quad \tilde{\varrho}\left(\tilde{\mathbf{v}}_{n j}\right)=e^{-\frac{2 \pi \gamma_{n j}}{\omega_{1}}}, \quad \tilde{\varrho}(\tilde{q})=e^{-\frac{2 \pi i \omega_{2}}{\omega_{1}}} .
$$


and the two actions of the torus and its dual on the same space of functions of $\gamma_{n i}$ mutually commute. Hence, we have the following

Proposition 3.2 The operators

$$
\begin{gathered}
\tilde{\varrho}\left(K_{n n}\right)=e^{-\frac{2 \pi}{\omega_{1}}\left(\sum_{j=1}^{n} \gamma_{n j}-\sum_{j=1}^{n-1} \gamma_{n-1, j}\right)}, \\
\tilde{\varrho}\left(E_{n, n+1}\right)=-\frac{2 i e^{-\frac{\pi i \omega_{2}}{\omega_{1}}(n-1)}}{\sin \frac{2 \pi \omega_{2}}{\omega_{1}}} \sum_{j=1}^{n} \frac{\prod_{r=1}^{n+1} \sinh \frac{2 \pi}{\omega_{1}}\left(\gamma_{n j}-\gamma_{n+1, r}-\frac{i \omega_{2}}{2}\right)}{\prod_{s \neq j} \sinh \frac{2 \pi}{\omega_{1}}\left(\gamma_{n j}-\gamma_{n s}\right)} e^{-i \omega_{2} \partial_{\gamma_{n j}}}, \\
\tilde{\varrho}\left(E_{n+1, n}\right)=\frac{i e^{\frac{\pi i \omega_{2}}{\omega_{1}}(n-1)}}{2 \sin \frac{2 \pi \omega_{2}}{\omega_{1}}} \sum_{j=1}^{n} \frac{\prod_{r=1}^{n-1} \sinh \frac{2 \pi}{\omega_{1}}\left(\gamma_{n j}-\gamma_{n-1, r}+\frac{i \omega_{2}}{2}\right)}{\prod_{s \neq j} \sinh \frac{2 \pi}{\omega_{1}}\left(\gamma_{n j}-\gamma_{n s}\right)} e^{i \omega_{2} \partial_{\gamma_{n j}}},
\end{gathered}
$$

generate a representation of the dual quantum group $U_{\tilde{q}}(\mathfrak{g l}(N))$, where $\tilde{q}=e^{-\frac{2 \pi i \omega_{2}}{\omega_{1}}}$. The operators $\varrho\left(U_{q}(\mathfrak{g l}(N))\right)$ and $\tilde{\varrho}\left(U_{\tilde{q}}(\mathfrak{g l}(N))\right)$ are commute by construction.

Thus we have a structure of a $U_{q}(\mathfrak{g l}(N)) \otimes U_{\tilde{q}}(\mathfrak{g l}(N))$-bimodule. More precisely, this bimodule may be characterized by the condition

$$
\varrho\left(K_{n n}\right)=\tilde{\varrho}\left(K_{n n}\right)^{-\tau}, \quad n=1, \ldots, N
$$

where $\tau=\omega_{1} / \omega_{2}$. We will discuss the better way to formulate this condition latter in this section.

Let us remark that we actually constructed the embeddings $\varrho: U_{q}(\mathfrak{g l}(N)) \hookrightarrow \mathcal{R}^{s}$ and $\tilde{\varrho}$ : $U_{\tilde{q}}(\mathfrak{g l}(N)) \hookrightarrow \mathcal{R}^{s}$. Consider the centralizer $\left[\mathcal{D}\left(\varrho\left(U_{q} \mathfrak{g l}(N)\right)\right)\right]^{\prime}$ of the algebras $\mathcal{D}\left(\varrho\left(U_{q} \mathfrak{g l}(N)\right)\right)$ as a subalgebra of $\mathcal{R}^{s}$. Then we have

\section{Theorem 3.2}

$$
\left[\mathcal{D}\left(\varrho\left(U_{q} \mathfrak{g l}(N)\right)\right)\right]^{\prime}=\mathcal{D}\left(\tilde{\varrho}\left(U_{\tilde{q}} \mathfrak{g l}(N)\right)\right) .
$$

This Theorem is proved by direct calculation.

Consider now more interesting case of $U_{q}(\mathfrak{s l}(N))$. It appears that the centralizer of the minimal (adjoint) rational form of the quantum group $U_{q}(\mathfrak{s l}(N))$ is described in terms of the different forms of the same quantum group. This may be considered as an indication of the fact that the Langlands dual of $P S L(N)$ is $S L(N)$. One may conjecture that the Langlands dual quantum group $U_{\tilde{q}}(\check{\mathfrak{g}})$ may be generally obtained as a result of explicit calculations of the centralizer in the appropriate generalization of the above construction to arbitrary quantum groups.

Below we give the explicit formulas for $U_{q}(\mathfrak{s l}(2))$. The adjoint rational form of the quantum group $U_{q}^{Q}(\mathfrak{s l}(2))$ is generated by elements $K, K^{-1}, E, F$ subjected to the relations

$$
\begin{gathered}
K E K^{-1}=q^{2} E, \quad K F K^{-1}=q^{-2} F, \\
E F-F E=\frac{K-K^{-1}}{q-q^{-1}} .
\end{gathered}
$$


The representation is given by

$$
\begin{aligned}
& \varrho(K)=e^{\frac{4 \pi \gamma_{11}}{\omega_{2}}}, \\
& \varrho(E)=\frac{2 i}{\sin \frac{2 \pi \omega_{1}}{\omega_{2}}} \sinh \frac{2 \pi}{\omega_{2}}\left(\gamma_{11}-\nu-\frac{i \omega_{1}}{2}\right) \sinh \frac{2 \pi}{\omega_{2}}\left(\gamma_{11}+\nu-\frac{i \omega_{1}}{2}\right) e^{-i \omega_{1} \partial \gamma_{11}} \text {, } \\
& \varrho(F)=-\frac{i}{\sin \frac{2 \pi \omega_{1}}{\omega_{2}}} e^{i \omega_{1} \partial \gamma_{11}} \\
& \varrho(q)=e^{\frac{2 \pi i \omega_{1}}{\omega_{2}}} .
\end{aligned}
$$

This representation is obtained from those of $U_{q}(\mathfrak{g l}(2))$ by the restriction $\gamma_{21}=-\gamma_{22}:=\nu$. The centralizer in $\mathcal{R}$ is generated by the algebra of functions of the dual torus $\mathbb{T}_{\tilde{q}^{1 / 2}}$ :

$$
\tilde{u} \tilde{v}=\tilde{q}^{1 / 2} \tilde{v} \tilde{u}
$$

where

$$
\tilde{\varrho}(\tilde{u})=e^{\frac{i \omega_{2}}{2} \partial \gamma_{11}}, \quad \tilde{\varrho}(\tilde{v})=e^{-\frac{2 \pi \gamma_{11}}{\omega_{1}}}, \quad \tilde{\varrho}(\tilde{q})=e^{-\frac{2 \pi i \omega_{2}}{\omega_{1}}} .
$$

The centralizer in $\mathcal{R}^{s}$ may be described as an image of the skew field of fractions of the following algebra generated by $\tilde{L}, \tilde{E}, \tilde{F}, \tilde{K}=\tilde{L}^{4}$ over the quadratic extension $\mathbb{C}\left(\tilde{q}^{1 / 2}\right)$ :

$$
\begin{gathered}
\tilde{L} \tilde{E} \tilde{L}^{-1}=\tilde{q}^{1 / 2} \tilde{E}, \quad \tilde{L} \tilde{F} \tilde{L}^{-1}=\tilde{q}^{-1 / 2} \tilde{F}, \\
\tilde{E} \tilde{F}-\tilde{F} \tilde{E}=\frac{\tilde{K}-\tilde{K}^{-1}}{\tilde{q}-\tilde{q}^{-1}} .
\end{gathered}
$$

under the representation

$$
\begin{aligned}
& \tilde{\varrho}(L)=e^{-\frac{2 \pi \gamma_{11}}{\omega_{1}}}, \\
& \tilde{\varrho}(E)=-\frac{2 i}{\sin \frac{2 \pi \omega_{2}}{\omega_{1}}} \sinh \frac{2 \pi}{\omega_{1}}\left(2 \gamma_{11}-\nu-\frac{i \omega_{2}}{2}\right) \sinh \frac{2 \pi}{\omega_{1}}\left(2 \gamma_{11}+\nu-\frac{i \omega_{2}}{2}\right) e^{-\frac{i \omega_{2}}{2} \partial \gamma_{11}}, \\
& \tilde{\varrho}(F)=\frac{i}{2 \sin \frac{2 \pi \omega_{2}}{\omega_{1}}} e^{\frac{i \omega_{2}}{2} \partial \gamma_{11}} .
\end{aligned}
$$

This algebra may be considered as a maximal form of $U_{\tilde{q}}(\mathfrak{s l}(2))$ over the quadratic extension $\mathbb{C}\left(\tilde{q}^{1 / 2}\right)$. Let us stress that the reconstruction of the algebra from its skew field of fractions is not unique. Thus, in this example the same centralizer may be interpreted as the image of the skew field of fractions of the simply-connected form $U_{\tilde{q}^{1 / 2}}^{P}(\mathfrak{s l}(2))$ :

$$
\begin{aligned}
& \tilde{\varrho}(L)=e^{-\frac{2 \pi \gamma_{11}}{\omega_{1}}}, \\
& \tilde{\varrho}(E)=-\frac{2 i}{\sin \frac{\pi \omega_{2}}{\omega_{1}}} \sinh \frac{2 \pi}{\omega_{1}}\left(\gamma_{11}-\nu-\frac{i \omega_{2}}{4}\right) \sinh \frac{2 \pi}{\omega_{1}}\left(\gamma_{11}+\nu-\frac{i \omega_{2}}{4}\right) e^{-\frac{i \omega_{2}}{2} \partial \gamma_{11}}, \\
& \tilde{\varrho}(F)=\frac{i}{2 \sin \frac{\pi \omega_{2}}{\omega_{1}}} e^{\frac{i \omega_{2}}{2} \partial \gamma_{11}} .
\end{aligned}
$$


where the operators satisfy the relations:

$$
\begin{gathered}
\tilde{L} \tilde{E} \tilde{L}^{-1}=\tilde{q}^{1 / 2} \tilde{E}, \quad \tilde{L} \tilde{F} \tilde{L}^{-1}=\tilde{q}^{-1 / 2} \tilde{F}, \\
\tilde{E} \tilde{F}-\tilde{F} \tilde{E}=\frac{\tilde{K}-\tilde{K}^{-1}}{\tilde{q}^{1 / 2}-\tilde{q}^{-1 / 2}},
\end{gathered}
$$

and $\tilde{K}=\tilde{L}^{2}$. Moreover, there is an isomorphism of the algebras $\mathcal{D}\left(U_{\tilde{q} 1 / 2}^{P}(\mathfrak{s l}(2))\right)$ and $\mathcal{D}\left(U_{\tilde{q} 1 / 4}^{Q}(\mathfrak{s l}(2))\right)$. This allows to reformulate the duality in a more symmetric form. Taking $p=e^{\pi i \tau}$ and $\tilde{p}=e^{-\pi i / \tau}$ with $\tau=\frac{2 \omega_{1}}{\omega_{2}}$ one gets the duality between algebra $U_{p}^{Q}(\mathfrak{s l}(2))$ and $U_{\tilde{p}}^{Q}(\mathfrak{s l}(2))$ which leads to the modular double considered in [13], [14]. Let us stress however that the dual quantum deformation parameters enter here in a non-standard way.

Finally, consider the algebra $U_{q}^{P}(\mathfrak{s l}(2))$ such that $\mathcal{D}\left(U_{q}^{P}(\mathfrak{s l}(2))\right)=\mathbb{T}_{q}:=\left\{v=e^{\frac{2 \pi \gamma_{11}}{\omega_{2}}}, u=\right.$ $\left.e^{i \omega_{1} \partial_{\gamma_{11}}}\right\}$ with $q=e^{\frac{2 \pi i \omega_{1}}{\omega_{2}}}$. Obviously, $\left[\mathcal{D}\left(U_{q}^{P}(\mathfrak{s l}(2))\right)\right]^{\prime}=\mathbb{T}_{\tilde{q}}:=\left\{\tilde{v}=e^{-\frac{2 \pi \gamma_{11}}{\omega_{1}}}, \tilde{u}=e^{i \omega_{2} \partial_{\gamma_{11}}}\right\}$ with $\tilde{q}=e^{-\frac{2 \pi i \omega_{2}}{\omega_{1}}}$. In other words, the algebras $\mathcal{D}\left(U_{q}^{P}(\mathfrak{s l}(2))\right)$ and $\mathcal{D}\left(U_{\tilde{q}}^{P}(\mathfrak{s l}(2))\right)$ with $q=e^{2 \pi i \tau}$ and $\tilde{q}=e^{-2 \pi i / \tau}, \tau=\frac{\omega_{1}}{\omega_{2}}$ centralize each other.

Clearly to use the centralizers as a way to describe the Langlands dual pairs deserves additional structures on the representation space comparing to what was discussed above. We are going to consider these matters elsewhere. Let us also remark that the use of the continuous powers of the Cartan generators in (3.21) is not quite appropriate in our setting. A possible solution is to identify instead the actions of the centers of both algebras. It can be shown that the center is described in terms of the symmetric polynomials of $\mathbf{v}_{N i}$ and thus the bimodule structure may be described equivalently as

$$
\varrho\left(\mathbf{v}_{N j}\right)=\tilde{\varrho}\left(\tilde{\mathbf{v}}_{N j}\right)^{-\tau} .
$$

If we consider $\log \mathbf{v}_{N j}$ as legitimate operators then the relations (3.31) make sense. However we believe that a proper description of the structure of this bimodule which solves this problem should be given in a different way. Let us notice that the description of the universal enveloping algebra in terms of the quantum tori has obvious asymmetry. The variables $\mathbf{v}_{N i}$ are coordinate functions on the commutative sub-torus and thus there is no natural definition of the dual torus through the centralizer. Thus it is natural to guess that some generalization of the universal enveloping algebra provides the proper setting for the discussion of the Langlands duality for quantum groups through the centralizers. The most natural candidate is the quantum group analogs $\mathcal{A}\left(G_{q}\right)$ of the differential operators on the group $\operatorname{Dif} f(G)$ and on the basic affine space $\operatorname{Dif} f(G / N)$. This leads to the interpretation of the resulting $\mathcal{A}\left(G_{q}\right) \otimes \mathcal{A}\left(\check{G}_{\tilde{q}}\right)$-bimodule as an explicit realization of the Morita equivalence of the algebras $\mathcal{A}\left(G_{q}\right)$ and $\mathcal{A}\left(\check{G}_{\tilde{q}}\right)$. Preliminary results based on the generalized Gelfand-Zetlin representation (see [27]) support this conjecture. We are going to discuss this approach in the future.

\subsection{Whittaker modules}

Let us define the special class of the representations of $U_{q}(\mathfrak{g l}(N)) \otimes U_{\tilde{q}}(\mathfrak{g l}(N))$, generalizing the Whittaker module for the classical algebras described in the previous section. The 
Whittaker vectors for $U_{q}(\mathfrak{g l}(N)) \otimes U_{\tilde{q}}(\mathfrak{g l}(N))$-bimodule are defined using the generalization of the definition in [15. ${ }^{2}$

Definition 3.1 The Whittaker vectors $w_{N}$ and $w_{N}^{\prime}$ are defined by equations

$$
\begin{aligned}
& E_{n, n+1} w_{N}=\frac{\chi_{n}}{q-q^{-1}} K_{n n} \prod_{m=1}^{N} K_{m m}^{c_{n m}-c_{n+1, m}} w_{N}, \\
& \tilde{E}_{n, n+1} w_{N}=\frac{\chi_{n}}{\tilde{q}-\tilde{q}^{-1}} \tilde{K}_{n n} \prod_{m=1}^{N} \tilde{K}_{m m}^{c_{n m}-c_{n+1, m}} w_{N}, \\
& E_{n+1, n} w_{N}^{\prime}=\frac{\chi_{n}^{\prime}}{q-q^{-1}} K_{n n}^{-1} \prod_{m=1}^{N} K_{m m}^{c_{n m}^{\prime}-c_{n+1, m}^{\prime}} w_{N}^{\prime}, \\
& \tilde{E}_{n+1, n} w_{N}^{\prime}=\frac{\chi_{n}^{\prime}}{\tilde{q}-\tilde{q}^{-1}} \tilde{K}_{n n}^{-1} \prod_{m=1}^{N} \tilde{K}_{m m}^{c_{n m}^{\prime}-c_{n+1, m}^{\prime}} w_{N}^{\prime},
\end{aligned}
$$

where $\left\|c_{n m}\right\|,\left\|c_{n m}^{\prime}\right\|$ are the $N \times N$ symmetric matrices such that $c_{n m}-c_{n+1, m}, c_{n m}^{\prime}-c_{n+1, m}^{\prime}$ are integers, and $\chi_{n}, \chi_{n}^{\prime}$ are arbitrary parameters.

The direct calculations show that the following statement is true:

Proposition 3.3 The defining equations (3.32), (3.39) are consistent with the full set of the Serre relations (3.2) and their dual analogues.

The results of Section 2 can be naturally extended to the quantum group case. The structure of the Whittaker vectors and Whittaker modules remains essentially the same. In particular, the Whittaker vectors can be written in a form similar to equations (2.8), (2.9). For example, there is a solution to (3.33) which is unique up to multiplication by an arbitrary double-periodic function:

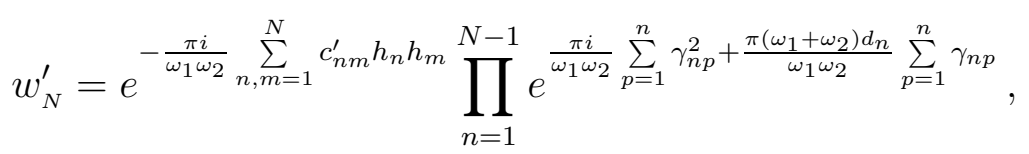

where

$$
h_{n}=\sum_{j=1}^{n} \gamma_{n j}-\sum_{j=1}^{n-1} \gamma_{n-1, j}
$$

and $d_{n}=2 n-c_{n n}^{\prime}+2 c_{n, n+1}^{\prime}-c_{n+1, n+1}^{\prime}-1$. In the present example $\chi_{n}^{\prime}=(-1)^{d_{n}}$.

Let us denote by $W_{N}^{\prime}$ the Whittaker module with the cyclic vector (3.34). It can be proved that it is spanned by the symmetric polynomials of the variables $e^{ \pm \frac{2 \pi \gamma_{n j}}{\omega_{1}}}, e^{ \pm \frac{2 \pi \gamma_{n j}}{\omega_{2}}}$ (compare with Section 2.2). The matrix elements of the particular group elements between the Whittaker vectors leads to the explicit expressions for the wave functions of the generalized quantum Toda theories and will considered elsewhere.

\footnotetext{
${ }^{2}$ For the case of $U_{q}(\mathfrak{g})$, where $\mathfrak{g}$ is an arbitrary simple Lie algebra, the construction of the non-degenerate characters of nilpotent subalgebras was done by Sevostyanov 28. However, the appearance of the bimodule structure reveals larger symmetry in the representation theory of quantum groups.
} 


\section{The QISM's eigenfunction via representation theory}

\subsection{An infinite dimensional representations of the Yangian}

The aim of this section is to introduce a special type of an infinite dimensional representations of the $Y(\mathfrak{g l}(N))$. This allows to connect the QISM methods of the solution of the integrable system based on the representation theory of Yangian and the solution based on the representation discussed in Section 2.

We start with some well known facts of the Yangian theory [11, 12] (see also the recent review [29]). The Yangian $Y(\mathfrak{g l}(N))$ is an associative Hopf algebra generated by the elements $T_{i j}^{(r)}$, where $i, j=1, \ldots, N$ and $r=0,1,2, \ldots$, subject the following relations. Consider the $N \times N$ matrix $T(\lambda)=\left\|T_{i j}(\lambda)\right\|_{i, j=1}^{N}$ with operator-valued entries

$$
T_{i j}(\lambda)=\lambda \delta_{i j}+\sum_{r=0}^{\infty} T_{i j}^{(r)} \lambda^{-r}
$$

Let

$$
R(\lambda)=I \otimes I+i \hbar P / \lambda, \quad P_{i k, j l}=\delta_{i l} \delta_{k j},
$$

be an $N^{2} \times N^{2}$ numerical matrix (the Yang $R$-matrix). Then the relations between the generators $T_{i j}^{(r)}$ can be written in the standard form

$$
R(\lambda-\mu)(T(\lambda) \otimes I)(I \otimes T(\mu))=(I \otimes T(\mu))(T(\lambda) \otimes I) R(\lambda-\mu) .
$$

The centre of the Yangian is generated by the coefficients of the formal Laurent series (the quantum determinant of $T(\lambda)$ in the sense of [4]):

$$
\begin{gathered}
\operatorname{det}_{q} T(\lambda) \\
=\sum_{s \in S_{N}} \operatorname{sign} s T_{s(1), 1}\left(\lambda-i \hbar \rho_{1}^{(N)}\right) \ldots T_{s(k), k}\left(\lambda-i \hbar \rho_{k}^{(N)}\right) \ldots T_{s(N), N}\left(\lambda-i \hbar \rho_{N}^{(N)}\right),
\end{gathered}
$$

where $\rho_{n}^{(N)}=\frac{1}{2}(N-2 n+1), n=1, \ldots, N$ and the summation is over the elements of the permutation group $S_{N}$. Let $X(\lambda)=\left\|X_{i j}(\lambda)\right\|_{i, j=1}^{n}$ be an $n \times n$ submatrix of the matrix $\left\|T_{i j}(\lambda)\right\|_{i, j=1}^{N}$. It is obvious from the explicit form of $R_{N}(\lambda)$ that this submatrix satisfies an analogue of relations (4.3). The quantum determinant $\operatorname{det}_{q} X(\lambda)$ is defined similarly to (4.4) (with the evident change $N \rightarrow n$ ).

The following way to describe the Yangian $Y(\mathfrak{g l}(N))$ was introduced in 11. Let $\mathbf{A}_{n}(\lambda)$, $n=1, \ldots, N$, be the quantum determinants of the submatrices, determined by the first $n$ rows and columns, and let the operators $\mathbf{B}_{n}(\lambda), \mathbf{C}_{n}(\lambda), n=1, \ldots, N-1$, be the quantum determinants of the submatrices with elements $T_{i j}(\lambda)$, where $i=1, \ldots, n ; j=1, \ldots, n-$ $1, n+1$ and $i=1, \ldots, n-1, n+1 ; j=1, \ldots, n$, respectively. The expansion coefficients of $\mathbf{A}_{n}(\lambda), \mathbf{B}_{n}(\lambda), \mathbf{C}_{n}(\lambda), n=1, \ldots, N-1$, with respect to $\lambda$, together with those of $\mathbf{A}_{N}(\lambda)$, 
generate the algebra $Y(\mathfrak{g l}(N))$. The parts of the relations, which we use below, are as follows:

$$
\begin{gathered}
{\left[\mathbf{A}_{n}(\lambda), \mathbf{A}_{m}(\mu)\right]=0 ; \quad(n, m=1, \ldots, N),} \\
{\left[\mathbf{B}_{n}(\lambda), \mathbf{B}_{m}(\mu)\right]=0 ; \quad\left[\mathbf{C}_{n}(\lambda), \mathbf{C}_{m}(\mu)\right]=0 ; \quad(m \neq n \pm 1),} \\
(\lambda-\mu+i \hbar) \mathbf{A}_{n}(\lambda) \mathbf{B}_{n}(\mu)=(\lambda-\mu) \mathbf{B}_{n}(\mu) \mathbf{A}_{n}(\lambda)+i \hbar \mathbf{A}_{n}(\mu) \mathbf{B}_{n}(\lambda), \\
(\lambda-\mu+i \hbar) \mathbf{A}_{n}(\mu) \mathbf{C}_{n}(\lambda)=(\lambda-\mu) \mathbf{C}_{n}(\lambda) \mathbf{A}_{n}(\mu)+i \hbar \mathbf{A}_{n}(\lambda) \mathbf{C}_{n}(\mu) .
\end{gathered}
$$

Let $A(\mathfrak{g l}(N))$ be the commutative subalgebra of $Y(\mathfrak{g l}(N))$ generated by $\mathbf{A}_{n}(\lambda), n=$ $1, \ldots, N$. It was proved in 31 that $A(\mathfrak{g l}(N))$ is the maximal commutative subalgebra of $Y(\mathfrak{g l}(N))$.

There is another realization of the $Y(\mathfrak{g l}(N))$ introduced by Drinfeld [12]. The algebra $Y(\mathfrak{g l}(N))$ is generated by the coefficients of the formal series

$$
\begin{gathered}
k_{n}(\lambda)=\lambda+\sum_{a=0}^{\infty} k_{n}^{(a)} \lambda^{-a} \\
e_{n}(\lambda)=\sum_{a=0}^{\infty} e_{n}^{(a)} \lambda^{-a-1} \\
f_{n}(\lambda)=\sum_{a=0}^{\infty} f_{n}^{(a)} \lambda^{-a-1}
\end{gathered}
$$

subjected to the commutation relations

$$
\begin{gathered}
{\left[k_{n}(\lambda), k_{m}(\mu)\right]=0} \\
{\left[k_{n}(\lambda), e_{m}(\mu)\right]=i \hbar\left(\delta_{n m}-\delta_{n, m+1}\right) k_{n}(\lambda) \frac{e_{m}(\lambda)-e_{m}(\mu)}{\lambda-\mu},} \\
{\left[k_{n}(\lambda), f_{m}(\mu)\right]=-i \hbar\left(\delta_{n m}-\delta_{n, m+1}\right) \frac{f_{m}(\lambda)-f_{m}(\mu)}{\lambda-\mu} k_{n}(\lambda),} \\
{\left[e_{n}(\lambda), f_{m}(\mu)\right]=i \hbar \frac{k_{n}^{-1}(\mu) k_{n+1}(\mu)-k_{n}^{-1}(\lambda) k_{n+1}(\lambda)}{\lambda-\mu} \delta_{n m},} \\
{\left[e_{n}^{(a+1)}, e_{m}^{(b)}\right]-\left[e_{n}^{(a)}, e_{m}^{(b+1)}\right]=\frac{\imath \hbar}{2} a_{n m}\left(e_{n}^{(a)} e_{m}^{(b)}+e_{m}^{(b)} e_{n}^{(a)}\right),} \\
{\left[f_{n}^{(a+1)}, f_{m}^{(b)}\right]-\left[f_{n}^{(a)}, f_{m}^{(b+1)}\right]=-\frac{\imath \hbar}{2} a_{n m}\left(f_{n}^{(a)} f_{m}^{(b)}+f_{m}^{(b)} f_{n}^{(a)}\right),} \\
{\left[e_{n}^{(a)},\left[e_{n}^{(b)}, e_{n \pm 1}^{(c)}\right]\right]+\left[e_{n}^{(b)},\left[e_{n}^{(b)}, e_{n \pm 1}^{(c)}\right]\right]=0,} \\
{\left[f_{n}^{(a)},\left[f_{n}^{(b)}, f_{n \pm 1}^{(c)}\right]\right]+\left[f_{n}^{(b)},\left[f_{n}^{(b)}, f_{n \pm 1}^{(c)}\right]\right]=0,}
\end{gathered}
$$

where $a_{n m}=2 \delta_{n m}-\delta_{n, m+1}-\delta_{n+1, n}$. The relation between two realization is given by

$$
\begin{gathered}
k_{n}(\lambda)=\frac{\mathbf{A}_{n}\left(\lambda-\frac{i(n-1) \hbar}{2}\right)}{\mathbf{A}_{n-1}\left(\lambda-\frac{i n \hbar}{2}\right)} \\
e_{n}(\lambda)=\mathbf{A}_{n}^{-1}\left(\lambda-\frac{i(n-1) \hbar}{2}\right) \mathbf{B}_{n}\left(\lambda-\frac{i(n-1) \hbar}{2}\right), \\
f_{n}(\lambda)=\mathbf{C}_{n}\left(\lambda-\frac{i(n-1) \hbar}{2}\right) \mathbf{A}_{n}^{-1}\left(\lambda-\frac{i(n-1) \hbar}{2}\right) .
\end{gathered}
$$


Let us stress that Drinfeld's realization is known for the $Y(\mathfrak{g})$, where $\mathfrak{g}$ be any simple Lie algebra [1]-[12].

There is a natural epimorphism $\pi_{N}: Y(\mathfrak{g l}(N)) \rightarrow U(\mathfrak{g l}(N))$

$$
\pi_{N}\left(T_{j k}(\lambda)\right)=\lambda \delta_{j k}-i \hbar E_{j k}, \quad(j, k=1, \ldots, N) .
$$

Denote the images under $\pi_{N}$ of the generators $\mathbf{A}_{n}(\lambda)$ and $\mathbf{B}_{n}(\lambda), \mathbf{C}_{n}(\lambda)$ by $\mathcal{A}_{n}(\lambda)$ and $\mathcal{B}_{n}(\lambda), \mathcal{C}_{n}(\lambda)$, respectively. Note that the images are the polynomials in $\lambda$ of orders $n$ and $n-1$, respectively.

To obtain the representation of the Yangian $Y(\mathfrak{g l}(N))$ we start with the construction of a natural representation of the Cartan subalgebra generated by $k_{n}(\lambda)$. It can be represented by the rational functions as follows:

$$
\prod_{s=1}^{n} k_{s}\left(\lambda-i \hbar \rho_{s}^{(n)}\right)=\prod_{j=1}^{n}\left(\lambda-\gamma_{n j}\right)
$$

where $\rho_{s}^{(n)}=\frac{1}{2}(n-2 s+1)$ and $n=1, \ldots, N$. Then the operators $A(\mathfrak{g l}(N))$ act by the polynomials $\mathcal{A}_{n}(\lambda)=\prod_{j=1}^{n}\left(\lambda-\gamma_{n j}\right), n=1, \ldots, N$. We resolve the rest of the Yangian relations and find the explicit expressions for the generators $e_{n}(\lambda), f_{n}(\lambda)\left(\right.$ and $\mathcal{B}_{n}(\lambda)$ and $\mathcal{C}_{n}(\lambda)$ ) in terms of the operators acting on the space of functions depending on the variables $\gamma_{n j}, j=$ $1, \ldots, n ; n=1, \ldots, N$.

Theorem 4.1 The operators

$$
\begin{gathered}
k_{n}(\lambda)=\frac{\prod_{j=1}^{n}\left(\lambda-\gamma_{n j}-\frac{i(n-1) \hbar}{2}\right)}{\prod_{j=1}^{n-1}\left(\lambda-\gamma_{n-1, j}-\frac{i n \hbar}{2}\right)}, \\
e_{n}(\lambda)=\sum_{j=1}^{n} \frac{1}{\lambda-\gamma_{n j}-\frac{i(n-1) \hbar}{2}} \frac{\prod_{r=1}^{n+1}\left(\gamma_{n j}-\gamma_{n+1, r}-\frac{i \hbar}{2}\right)}{\prod_{s \neq j}\left(\gamma_{n j}-\gamma_{n s}\right)}, \\
f_{n}(\lambda)=\sum_{j=1}^{n} \frac{1}{\lambda-\gamma_{n j}-i \hbar-\frac{i(n-1) \hbar}{2}} \frac{\prod_{r=1}^{n-1}\left(\gamma_{n j}-\gamma_{n-1, r}+\frac{i \hbar}{2}\right)}{\prod_{s \neq j}\left(\gamma_{n j}-\gamma_{n s}\right)} e^{i \hbar \partial_{\gamma_{n j}}},
\end{gathered}
$$

satisfy the complete set of relations (4.7) and, therefore, define a representation of the Yangian $Y(\mathfrak{g l}(N))$. 
As a consequence we have

$$
\begin{gathered}
\mathcal{A}_{n}(\lambda)=\prod_{j=1}^{n}\left(\lambda-\gamma_{n j}\right), \\
\mathcal{B}_{n}(\lambda)=\sum_{j=1}^{n} \prod_{s \neq j} \frac{\lambda-\gamma_{n s}}{\gamma_{n j}-\gamma_{n s}} \prod_{r=1}^{n+1}\left(\gamma_{n j}-\gamma_{n+1, r}-\frac{i \hbar}{2}\right) e^{-i \hbar \partial_{\gamma_{n j}}} \\
\mathcal{C}_{n}(\lambda)=-\sum_{j=1}^{n} \prod_{s \neq j} \frac{\lambda-\gamma_{n s}}{\gamma_{n j}-\gamma_{n s}} \prod_{r=1}^{n-1}\left(\gamma_{n j}-\gamma_{n-1, r}+\frac{i \hbar}{2}\right) e^{i \hbar \partial_{\gamma_{n j}}}
\end{gathered}
$$

Note that we also obtain the representation discussed in Section 2 through the following

integral formulas which express the generators $E_{i j}$ of the Lie algebra $\mathfrak{g l}(N)$ in terms of the Yangian generators

$$
\begin{gathered}
E_{n, n+1}=\frac{1}{2 \pi \hbar} \oint e_{n}(\lambda) d \lambda,(n=1, \ldots, N-1), \\
E_{n+1, n}=\frac{1}{2 \pi \hbar} \oint f_{n}(\lambda) d \lambda,(n=1, \ldots, N-1), \\
E_{n n}=\frac{1}{2 \pi \hbar} \oint k_{n}(\lambda) \frac{d \lambda}{\lambda}-\frac{1}{2}(n-1),(n=1, \ldots, N) .
\end{gathered}
$$

The non-simple root generators are defined recursively as $E_{j k}=\left[E_{j m}, E_{m k}\right]$ for $j<m<k$ and $j>m>k$. Here, the integrands are understood as Laurent series and the contours of integrations are taken around $\infty$.

Let us finally remark, that there is a direct generalization of the Yangian to the case of quantum group [30] and it is possible extend the results of this section to the quantum group case.

\subsection{The Toda chains and $R$-matrix formalism}

The quantum Toda chain is one of the popular examples of integrable system. It is described by the Hamiltonian

$$
H=\sum_{n=1}^{N}\left(\frac{p_{n}^{2}}{2}+e^{x_{n}-x_{n+1}}\right),
$$

where $\left[x_{n}, p_{m}\right]=i \hbar \delta_{n m}$. There are two different ways to fix the boundary conditions: The choice of $x_{N+1}=\infty$ corresponds to the open $(G L(N))$ Toda chain; while the choice of $x_{N+1}=x_{1}$ corresponds to the periodic (affine) Toda chain.

The open and periodic Toda chains can be described, uniformly, by using the $R$ - matrix formalism [32]. Introduce the Lax operators

$$
L_{n}(\lambda)=\left(\begin{array}{cc}
\lambda-p_{n} & e^{-x_{n}} \\
-e^{x_{n}} & 0
\end{array}\right), \quad n=1, \ldots, N,
$$


satisfying the following commutation relations

$$
\left.R(\lambda-\mu)\left(L_{n}(\lambda)\right) \otimes I\right)\left(I \otimes L_{m}(\mu)\right)=\left(I \otimes L_{m}(\mu)\right)\left(L_{n}(\lambda) \otimes I\right) R(\lambda-\mu) \delta_{n m},
$$

with the rational $4 \times 4 R$-matrix

$$
R(\lambda)=I \otimes I+\frac{i \hbar}{\lambda} P
$$

The monodromy matrix

$$
T_{N}(\lambda)=L_{N}(\lambda) \ldots L_{1}(\lambda):=\left(\begin{array}{cc}
A_{N}(\lambda) & B_{N}(\lambda) \\
C_{N}(\lambda) & D_{N}(\lambda)
\end{array}\right)
$$

satisfies the equation

$$
R(\lambda-\mu)(T(\lambda) \otimes I)(I \otimes T(\mu))=(I \otimes T(\mu))(T(\lambda) \otimes I) R(\lambda-\mu) .
$$

In particular, the following commutation relations hold:

$$
\begin{gathered}
{\left[A_{N}(\lambda), A_{N}(\mu)\right]=\left[C_{N}(\lambda), C_{N}(\mu)\right]=0,} \\
(\lambda-\mu+i \hbar) A_{N}(\mu) C_{N}(\lambda)=(\lambda-\mu) C_{N}(\lambda) A_{N}(\mu)+i \hbar A_{N}(\lambda) C_{N}(\mu) .
\end{gathered}
$$

From (4.19) it can be easily shown that the trace of the monodromy matrix

$$
\widehat{t}_{N}(\lambda)=A_{N}(\lambda)+D_{N}(\lambda)
$$

satisfies $[\widehat{t}(\lambda), \widehat{t}(\mu)]=0$ and is a generating function for the Hamiltonians of the periodic Toda chain:

$$
\widehat{t}_{N}(\lambda)=\sum_{k=0}^{N}(-1)^{k} \lambda^{N-k} H_{k} .
$$

We formulate the spectral problems for periodic Toda chain as follows:

$$
\widehat{t}_{N}(\lambda) \Psi_{\boldsymbol{E}}=t_{N}(\lambda ; \boldsymbol{E}) \Psi_{\boldsymbol{E}},
$$

where

$$
t_{N}(\lambda ; \boldsymbol{E})=\sum_{k=0}^{N}(-1)^{k} \lambda^{N-k} E_{k} .
$$

On the other hand, the operator

$$
A_{N}(\lambda):=\sum_{k=0}^{N}(-1)^{k} \lambda^{N-k} h_{k}
$$

is a generating function of the Hamiltonians $h_{k}$ of the $N$ particles open Toda chain. The generating functions for the open Toda chains are connected by the recursive relations:

$$
\begin{gathered}
A_{N}(\lambda)=\left(\lambda-p_{N}\right) A_{N-1}(\lambda)+e^{-x_{N}} C_{N-1}(\lambda), \\
C_{N}(\lambda)=-e^{x_{N}} A_{N-1}(\lambda) .
\end{gathered}
$$




\subsection{The spectral problem for the open Toda chain}

The main goal of this subsection is to apply the results from the section 2 , to solution of the spectral problem of the open Toda chain.

Denote $\boldsymbol{x}=\left(x_{1}, \ldots, x_{N}\right)$. To solve the spectral problem we should find the common eigenfunction of the system of differential-difference equations:

$$
\begin{gathered}
A_{N}(\lambda) \psi_{\boldsymbol{\gamma}_{N}}(\boldsymbol{x})=\prod_{m=1}^{N}\left(\lambda-\gamma_{N m}\right) \psi_{\boldsymbol{\gamma}_{N}}(\boldsymbol{x}), \\
A_{N-1}\left(\gamma_{N j}\right) \psi_{\boldsymbol{\gamma}_{N}}(\boldsymbol{x})=i^{1-N} e^{-x_{N}} e^{-i \hbar \partial_{\gamma_{N j}}} \psi_{\boldsymbol{\gamma}_{N}}(\boldsymbol{x}),
\end{gathered}
$$

$j=1, \ldots, N$. It is worth mentioning that the system (4.27) is the quantum counterpart of the Flashka and McLaughlin [33] Darboux transform to separated variables $(p, x) \rightarrow(\gamma, \theta)$. For the first time the system (4.27) was introduced and solved in the framework of QISM (22]). Below we describe the representation theory solution of the system (4.27).

Let $W^{\prime}$ and $W$ be the dual irreducible Whittaker modules and $w_{N}^{\prime} \in W^{\prime}, w_{N} \in W$ be the corresponding cyclic Whittaker vectors. The representation of the Cartan subalgebra is integrated to the action of the Cartan torus, so the following function is well defined

$$
\psi_{\gamma_{N 1}, \ldots, \gamma_{N N}}=e^{-\boldsymbol{x} \cdot \boldsymbol{\rho}^{(N)}}\left\langle w_{N}^{\prime}, e^{-\sum_{k=1}^{N} x_{k} E_{k k}} w_{N}\right\rangle
$$

where $\boldsymbol{x} \cdot \boldsymbol{\rho}^{(N)}$ is the standard product in $\mathbb{R}^{N}$.

Definition 4.1 The radial projections $A_{n}(\lambda)$ of the generation functions $\mathcal{A}_{n}(\lambda)$ (2.13) of the central elements of $\mathcal{U}(\mathfrak{g l}(N))$ are defined by

$$
A_{n}(\lambda) \psi_{\gamma_{N 1}, \ldots, \gamma_{N N}}=e^{-\boldsymbol{x} \cdot \boldsymbol{\rho}^{(N)}}\left\langle w_{N}^{\prime}, e^{-\sum_{k=1}^{N} x_{k} E_{k k}} \mathcal{A}_{n}\left(\lambda-\frac{i(N-n) \hbar}{2}\right) w_{N}\right\rangle
$$

There is the relation between the operators $A_{n}(\lambda)$ of different levels:

$$
A_{n}(\lambda)=\left(\lambda-p_{n}\right) A_{n-1}(\lambda)-e^{x_{n-1}-x_{n}} A_{n-2}(\lambda),
$$

where $n=1, \ldots, N$ and $A_{-1}=0, A_{0}=1$. Therefore the $A_{n}(\lambda)$ is the generation function of the Hamiltonians of the $n$ - particles open Toda chain.

The following theorem identifies our construction of the matrix element (4.28) with the integral formula for the eigenfunction of the open Toda chain in terms of the Mellin-Barnes integrals 22$]$.

Theorem 4.2 The matrix element 4.28) satisfies the set of equations (4.27).

It remains to express the matrix element (4.28) in the integral form. Substituting the 
expressions (2.8), (2.9), and (2.2a) into (4.28), we obtain

$$
\begin{gathered}
\psi_{\boldsymbol{\gamma}_{N}}(\boldsymbol{x})=e^{-\boldsymbol{x} \cdot \boldsymbol{\rho}^{(N)}} \\
\times \int_{\mathbb{R}} \prod_{n=1}^{N-1} \frac{\prod_{k=1}^{n} \prod_{m=1}^{n+1} \hbar^{\frac{\gamma_{n k}-\gamma_{n+1, m}}{i \hbar}+\frac{1}{2}} \Gamma\left(\frac{\gamma_{n k}-\gamma_{n+1, m}}{i \hbar}+\frac{1}{2}\right)}{\prod_{s<p}\left|\Gamma\left(\frac{\gamma_{n s}-\gamma_{n p}}{i \hbar}\right)\right|^{2}} e^{\frac{i}{\hbar} \sum_{n, j=1}^{N}\left(\gamma_{n j}-\gamma_{n-1, j}\right) x_{n}} \prod_{\substack{n=1 \\
j \leq n}}^{N-1} d \gamma_{n j},
\end{gathered}
$$

where by definition $\gamma_{n j}=0$ for $j>n$.

In the study of the analytic properties of this solution with respect to $\gamma_{N}$, it is useful to transform (4.31). Let us change the variables of integration in (4.31):

$$
\gamma_{n j} \rightarrow \gamma_{n j}-\frac{i \hbar}{n} \sum_{s=1}^{n} \rho_{s}^{(N)}, \quad(n=1, \ldots, N-1)
$$

After the change of variables (4.32) we shift the domain of integration $\mathbb{R}^{\frac{N(N-1)}{2}}$ to the complex plane in such a way that the domain of integration over the variables $\gamma_{n-1, j}$ lies above the domain of integration over the variables $\gamma_{n j}$. Thus, we arrive at the analytic continuation equal to:

$$
\begin{gathered}
\psi_{\boldsymbol{\gamma}_{N}}\left(x_{1}, \ldots, x_{N}\right) \\
=\int_{\mathcal{C}} \prod_{n=1}^{N-1} \frac{\prod_{k=1}^{n} \prod_{m=1}^{n+1} \hbar^{\frac{\gamma_{n k}-\gamma_{n+1, m}}{i \hbar}} \Gamma\left(\frac{\gamma_{n k}-\gamma_{n+1, m}}{i \hbar}\right)}{\prod_{s \neq p} \Gamma\left(\frac{\gamma_{n s}-\gamma_{n p}}{i \hbar}\right)} e^{\frac{i}{\hbar} \sum_{n, j=1}^{N}\left(\gamma_{n j}-\gamma_{n-1, j}\right) x_{n}} \prod_{\substack{n=1 \\
j \leq n}}^{N-1} d \gamma_{n j},
\end{gathered}
$$

where the domain of integration $\mathcal{C}$ is defined by the conditions

$$
\min _{j}\left\{\operatorname{Im} \gamma_{k j}\right\}>\max _{m}\left\{\operatorname{Im} \gamma_{k+1, m}\right\}
$$

for all $k=1, \ldots, N-1$. The integral (4.33) converges absolutely. Thus, we obtain the integral representation 22] for the eigenfunction of open Toda chain by purely representation theory methods.

Finally, let us note that the Gelfand-Zetlin type representation may be generalized to the case of the $Y(\mathfrak{g})$, with $\mathfrak{g}$ being an arbitrary simple Lie algebra. This provides the uniform approach to the solution of the various integrable systems based on various (quantum) Lie groups. We are planning to discuss these results elsewhere.

\section{References}

[1] V.G. Drinfeld, Hopf algebras and the quantum Yang-Baxter equation, Soviet Math. Dokl. 32, 254-258, (1985). 
[2] M. Jimbo, A q-difference analogue of $U(\mathfrak{g})$ and the Yang-Baxter equation, Lett. Math. Phys. 10, (1985), 63-69.

[3] L.D. Faddeev, Quantum completely integrable models in field theory, Sov. Sci. Rev., Sect. C (Math. Phys. Rev.) 1, (1980), 107-155.

[4] P.P. Kulish, E.K. Sklyanin, Quantum spectral transform method. Recent developments, Lecture Notes in Phys. 151, pp. 61-119, Springer, Berlin-New York, 1982.

[5] E.K. Sklyanin, Separation of variables - new trends, Quantum field theory, integrable models and beyond, (Kyoto, 1994). Progr. Theor. Phys. Suppl. 118, (1995), 35-60.

[6] A. Gerasimov, S. Kharchev, D. Lebedev, Representation Theory and Quantum Inverse Scattering Method: The Open Toda Chain and the Hyperbolic Sutherland Model, Int. Math. Res. Notices 2004, no.17, (2004), 823-854.

[7] A. Gerasimov, S. Kharchev, D. Lebedev, On a class of integrable systems connected with $G L(N, \mathbb{R})$, arXiv:math.QA/0301025.

[8] I.M. Gelfand, M.L. Tsetlin, Finite-dimensional representations of the group of unimodular matrices, Dokl. Akad. Nauk SSSR 71, (1950), 825-828.

[9] I.M. Gelfand, M.I. Graev, Finite-dimensional irreducible representations of the unitary and the full linear groups, and related special functions, Izv. Akad. Nauk SSSR, Ser. Mat. 29, (1965), 1329-1356; [Transl., II Ser., Am. Math. Soc. 64, (1965), 116-146].

[10] F. Lemire, J. Patera, Formal analytic continuation of Gelfand's finite-dimensional representations of $g l(n, C)$, J. Math. Phys. 20, (1979), 820-829.

[11] V.G. Drinfeld, Quantum groups, Proc. Int. Congr. Math. Berkeley, California, (1986), Providence (1987), 718-820.

[12] V.G. Drinfeld, A new realization of Yangians and of quantum affine algebras, (Russian) Dokl. Akad. Nauk SSSR 296, (1987), no. 1, 13-17; translation in Soviet Math. Dokl. 36, (1988), 212-216.

[13] L. D. Faddeev, Discrete Heisenberg-Weyl group and modular group, Lett. Math. Phys. 34, (1995), 249-254.

[14] L. D. Faddeev, Modular double of a quantum group, in: Conférence Moshé Flato 1999, Quantization, Deformations, and Symmetries. Vol. I, 149-156, Kluwer Acad. Publ., Dordrecht, 2000.

[15] S. Kharchev, D. Lebedev, M. Semenov-Tian-Shansky, Unitary representations of $U_{q}(\mathfrak{s l}(2, \mathbb{R}))$, the modular double and the multiparticle q-deformed Toda chains, Comm. Math. Phys. 225, (2002), 573-609.

[16] G. Lusztig, Introduction to quantum groups, Progress in Mathematics, 110, Birkhäuser Boston, Inc., Boston, MA, 1993 
[17] V. Chari, A. Pressley, A guide to quantum groups, Cambridge Univ. Press, Cambridge, 1994.

[18] A. Connes, Non commutative geometry, Academic Press, 1994.

[19] M. Rieffel, $C^{*}$-algebras associated with irrational rotations, Pacific J. Math. 93, (1981), $415-430$.

[20] J. Adams, D. Barbasch and D. Vogan Jr. The Langlands Classification and Irreducible Chracters for Real Reductive Groups, Progress in Mathematics 104, Birkhäuser 1992.

[21] S. Kharchev, D. Lebedev, Integral representation for the eigenfunctions of a quantum periodic Toda chain, Lett. Math. Phys. 50, (1999), 53-77.

[22] S. Kharchev, D. Lebedev, Eigenfunctions of $G L(N, \mathbb{R})$ Toda chain: The Mellin-Barnes representation, JETP Lett. 71, (2000), 235-238.

[23] S. Kharchev, D. Lebedev, Integral representations for the eigenfunctions of quantum open and periodic Toda chains from QISM formalism, J.Phys. A34, (2001), 2247-2258.

[24] B. Kostant, On Whittaker vectors and representation theory, Inventiones Math. 48, (1978), 101-184.

[25] R. Howe, T. Umeda, The Capelli identity, the double commutant theorem, and multiplicity-free actions, Math. Ann. 290, (1991), 569-619.

[26] I. Gelfand, A. Kirillov, Sur les corps liés aux algèbres enveloppantes des algèbres de Lie, Publ. Math. de l'IHÉS 31, (1966), 509-523.

[27] A. Gerasimov, S. Kharchev, D. Lebedev, in preparation.

[28] A. Sevostyanov, Regular nilpotent elements and quantum groups, Comm. Math. Phys. 204, (1999), 1-16.

[29] A. Molev, M. Nazarov, G. Olshanski, Yangians and classical Lie algebras, Russian Math. Surveys 51, (1996), 205-282.

[30] M. Nazarov, V. Tarasov, Yangians and Gelfand-Zetlin bases, Publ. Res. Inst. Math. Sci. 30, (1994), 459-478.

[31] I.V. Cherednik, A new interpretation of Gelfand-Tzetlin bases, Duke Math. J. 54, (1987), 563-577.

[32] E.K. Sklyanin, The quantum Toda chain, Lect. Notes in Phys. 226, (1985), 196-233.

[33] H. Flaschka, D. McLaughlin, Canonically conjugate variables for the Korteweg-de Vries equation and the Toda lattice with periodic boundary conditions, Progr. Theor. Phys. 55, (1976), 438-456. 